\title{
Lattice BGK simulations of flow in a symmetric bifurcation
}

\author{
A.M. Artoli ${ }^{\text {a,* }}$, D. Kandhai ${ }^{\text {b }}$, H.C.J. Hoefsloot ${ }^{\text {c }}$, A.G. Hoekstra ${ }^{\text {a }}$, P.M.A. Sloot ${ }^{\text {a }}$ \\ a Section Computational Science, Faculty of Science, Laboratory for Computing, Systems Architecture and Programming, \\ University of Amsterdam, Kruislaan 403, 1098 SJ Amsterdam, The Netherlands \\ ${ }^{\mathrm{b}}$ Faculty of Applied Sciences, Kramers Laboratorium voor Fysische Technologie, Delft, The Netherlands \\ ${ }^{\mathrm{c}}$ Department of Chemical Engineering, Faculty of Science, University of Amsterdam, Amsterdam, The Netherlands
}

Available online 20 February 2004

\begin{abstract}
Surgical planning as a treatment for vascular diseases requires fast blood flow simulations that are efficient in handling changing geometry. It is, for example, necessary to try different paths of a planned bypass and study the resulting hemodynamic flow fields before deciding the final geometrical solution. With the aid of a real time interactive simulation environment that uses an efficient flow solver, this allows flexible treatment planning. In this article, we demonstrate that the lattice Boltzmann method can be an alternative robust computational fluid dynamics technique for such kind of applications. Steady flow in a 2D symmetric bifurcation is studied and the obtained flow fields and stress tensor components are compared to those obtained by a Navier-Stokes (NS) solver. We also demonstrate that the method is fully adaptive to interactively changing geometry.

(C) 2003 Elsevier B.V. All rights reserved.
\end{abstract}

Keywords: Symmetric bifurcation; Changing geometry; Lattice Boltzmann; Finite volume; Hemodynamics

\section{Introduction}

Flow characteristics near branches and bifurcations are quite important in hemodynamics. Cardiovascular diseases, a leading cause of mortality in the western world [17], localize in segments of the arterial system where the shear stress is low. Frequently, treatment of such diseases may involve planning for a new host artery or design of suitable cardiovascular devices, which are complex and patient specific.

Recently, two major developments in the field of vascular surgery planning have made it possible to

\footnotetext{
* Corresponding author.

E-mail addresses: artoli@science.uva.nl (A.M. Artoli),

b.d.kandhai@tnw.tudelft.nl (D. Kandhai), huubh@science.uva.nl

(H.C.J. Hoefsloot), alfons@ @science.uva.nl

(A.G. Hoekstra), sloot@science.uva.nl (P.M.A. Sloot).

URL: http://www.science.uva.nl/sloot/.
}

better and faster plan risk reduced surgeries. Firstly, magnetic resonance angiography (MRA) has been considerably enhanced to provide excellent and fast depiction of the arterial tree and non-invasive dynamic data acquisition is made possible [9]. Secondly, the development of cheap computing power and interactive simulation environments have made real time simulations of blood flow not far from reach $[8,18]$. With these in hand, an efficient and robust flow solver has to be used in an interactive modeling environment [1]. The field of computational fluid dynamics (CFD) has also developed extensively during the last two decades. New particle based methods such as dissipative particle dynamics, lattice gases and lattice Boltzmann methods have been developed and matured. The lattice Boltzmann method with Bhatnagar, Gross and Krook (BGK) simplified collision operator is a discretization of a discrete velocity Boltzmann equa- 
tion that describes the evolution of particles in kinetic theory. Due to its simple implementation, straightforward parallelization and easy grid generation, the capability of the lattice Boltzmann method has been demonstrated in various applications including Newtonian blood flow simulations [10], non-Newtonian and suspension flows [11]. Throughout the rest of this paper, we present the capability of the lattice Boltzmann method as a robust technique for interactive blood flow simulations by considering the case of a photo-typical symmetric bifurcation with a changing geometry.

\section{The lattice Boltzmann method}

Different from the traditional CFD methods which obtain the velocity and pressure by solving the Navier-Stokes equations and computing the shear stress from the velocity profiles, the lattice Boltzmann method is a special finite difference discretization of the simplified Boltzmann equation with BGK collision operator $[2,6,15]$ which describes transport phenomena at the mesoscale level. The dynamics of the fluid is modeled by the transport of simple fictitious particles on the nodes of a Cartesian grid. Simulations with this method involve two simple steps; streaming to the neighboring nodes and colliding with local node populations represented by the probability $f_{i}$ of a particle moving with a velocity $\mathbf{e}_{i}$ per unit time step $\delta t$. Populations are relaxed towards their equilibrium states during a collision process. The equilibrium distribution function

$$
f_{i}^{(e q)}=w_{i} \rho\left(1+\frac{3}{v^{2}} \mathbf{e}_{i} \cdot \mathbf{u}+\frac{9}{2 v^{4}}\left(\mathbf{e}_{i} \cdot \mathbf{u}\right)^{2}-\frac{3}{2 v^{2}} \mathbf{u} \cdot \mathbf{u}\right)
$$

is a low Mach number approximation to the Maxwellian distribution. Here, $w_{i}$ is a weighting factor, $v=\delta x / \delta t$ is the lattice speed, and $\delta x$ and $\delta t$ are the lattice spacing and the time step, respectively. Values for the weighting factor and the discrete velocities depend on the used lattice Boltzmann model and can be found in literature [2,6,19]. The lattice Boltzmann equation

$$
\begin{aligned}
& f_{i}\left(\mathbf{x}+\mathbf{e}_{i} \delta t, \mathbf{e}_{i}, t+\delta t\right)-f_{i}\left(\mathbf{x}, \mathbf{e}_{i}, t\right) \\
& \quad=-\frac{1}{\tau}\left[f_{i}\left(\mathbf{x}, \mathbf{e}_{i}, t\right)-f_{i}^{(0)}\left(\mathbf{x}, \mathbf{e}_{i}, t\right)\right],
\end{aligned}
$$

can be obtained by discretizing the evolution equation of the distribution functions in the velocity space using a finite set of velocities $\mathbf{e}_{i}$. In this equation, $\tau$ is the dimensionless relaxation time. By Taylor expansion of the lattice Boltzmann equation up to $\mathrm{O}\left(\delta t^{2}\right)$ and application of the multi-scale Chapman-Enskog technique [6], the Navier-Stokes equations and the momentum flux tensor up to second order in the Knudsen number are obtained. The hydrodynamic density, $\rho$, and the macroscopic velocity, $\mathbf{u}$, are determined in terms of the particle distribution functions from the laws of conservation of mass and momentum: $\rho=\sum_{i} f_{i}=$ $\sum_{i} f_{i}^{(e q)}$ and $\rho \mathbf{u}=\sum_{i} \mathbf{e}_{i} f_{i}=\sum_{i} \mathbf{e}_{i} f_{i}^{(e q)}$. The pressure is given by $p=\rho c_{\mathrm{s}}^{2}$ and the kinematic viscosity is $v=c_{\mathrm{S}}^{2} \delta t(\tau-1 / 2)$, where $c_{\mathrm{s}}$ is the speed of sound. Different lattice Boltzmann models differ in the choice of the distribution functions, the number of moving particles and the speed of sound inside the system. In our study, we have used an improved incompressible D2Q9i (two-dimensional, nine particles, incompressible) model [19], which has three types of particles on each node; a rest particle, four particles moving along $x$ and $y$ principal directions with speeds $\left|\mathbf{e}_{i}\right|= \pm 1$, and four particles moving along diagonal directions with speeds $\left|\mathbf{e}_{i}\right|=\sqrt{2}$. The stress tensor can be computed from the non-equilibrium parts of the distribution functions

$\sigma_{\alpha \beta}=-\rho c_{\mathrm{s}}^{2} \delta_{\alpha \beta}-\left(1-\frac{1}{2 \tau}\right) \sum_{i=0} f_{i}^{(1)} e_{i \alpha} e_{i \beta}$,

which is independent of the velocity fields, in contrast to the NS solvers for which a need to get the derivatives of obtained velocity profiles is not avoidable.

\section{Simulations}

We have carried out two different benchmark simulations. The first benchmark considers steady flow in a symmetric bifurcation and validates the results against a conventional NS solver. The second benchmark uses this bifurcation to study the robustness and response of the lattice Boltzmann method to a changing geometry. The general aim of these benchmark simulations is to demonstrate that the lattice Boltzmann yields accurate and robust results for applications related to hemodynamics and biomedical engineering. The two benchmarks are discussed below. 


\subsection{Steady flow in a symmetric bifurcation}

We are interested in the symmetric bifurcation as a $2 \mathrm{D}$ simplified model for arteries. As we mentioned above, there is a direct relation between the shear stress and Atherosclerosis, which is a highly localized disease in areas of the carotid, coronary and femoral arteries and abdominal aorta. All these locations have complex geometry, such as branching and bifurcation, complex flow patterns, secondary flow and complex shear stress. Several numerical and experimental models of fluid flow in large arteries and bifurcating tubes were previously studied $[3,12-14,16]$. However, in all these, authors used NS solvers to obtain the flow fields and approximated velocity gradients to get the shear stress. Studying the symmetric bifurcation as a benchmark for blood flow problems gives us a clearer idea about the complexity of the flow field and the shear stresses at locations of interest and allows us to investigate implementation of several boundary conditions before using them for more complex geometry.

In this study, we consider a simplified model of a two-dimensional symmetric bifurcation that consists of one main tube of diameter $D$ and length $L$ and two branches at the end of the main tube, each of which has an outer length $L$ and diameter $a=D / 2$. The angle $\theta$ between each branch and the centerline $\mathrm{AB}$ that passes horizontally across the divider is $30^{\circ}$. The geometry of the symmetric bifurcation is illustrated in Fig. 1, in which the centerline $\mathrm{AB}$ and the cross line $C D$ will be reference axes for measurements and comparisons of the flow fields and the components of

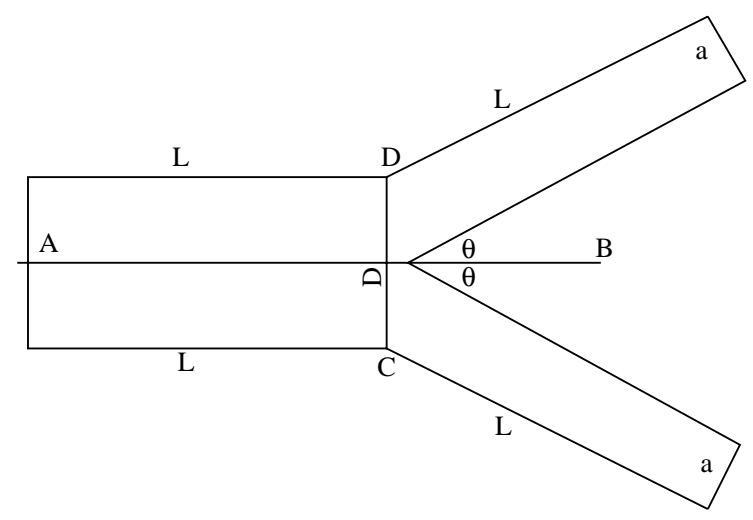

Fig. 1. Geometry of the simulated symmetric bifurcation. the stress tensor. The total vascular bed (i.e. the total cross-section) and consequently the average velocity of this bifurcation does not change in the daughter branches than those in the mother branch [13]. The geometry of the symmetric bifurcation whose vascular bed does not change, makes the region just before the divider an expanding region. This additional area has to be filled by the fluid. As a result, both the pressure and the velocity drop near to the divider before they enter the branches, where the velocity accelerates towards the fully developed flow and the pressure drops faster than the pressure in the main branch.

We have carried out a number of lattice Boltzmann simulations for steady flow in the symmetric bifurcation at $R e=1,200$ and 1250, where $R e=D U_{0} / v$ is the Reynolds number. The diameter of the main branch is represented by 40 lattice points on the coarsest grid and 320 lattice points on the finest grid. At the inlet, we have set a flat velocity $U_{0}$ of magnitude corresponding to the required Reynolds number. Once the velocity is known at the inlet, the pressure and the unknown values of the incoming (to the fluid) particle distributions can be computed from the outgoing (to the inlet) distribution functions in a simple way [20]. For the outlets, we have assumed fully developed flow. Finally, for the walls, we have implemented a simple bounce back scheme in which particles hitting the walls simply reverse their direction towards the fluid. Again a number of more accurate schemes are available to satisfy the non-slip condition, but the bounce back rule seems good enough for this benchmark and is more suitable for complex arterial systems, since it is adaptive, simple and fast.

The velocity profiles and the shear stress as obtained by LBM are shown in Fig. 2 for the three Reynolds numbers. As shown from these figures, the flow fully develops just after the inlet region for $R e=1$. However, for the larger Reynolds numbers, the flow is not yet fully developed when entering the divider region. At the outlets, the flow is fully developed for $R e=1$ and 200. For $R e=1250$, it appears that the flow is not fully developed at the outlets, but this does not have significant effects on the flow near to the divider (test simulations of varying lengths of the branches did not show significant difference (data not shown)). We also observe that the flow near the divider becomes 


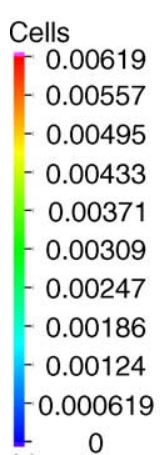

(a)

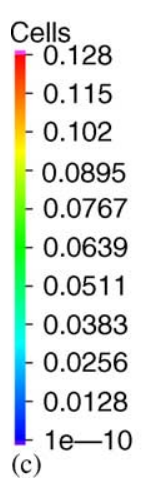

(c)

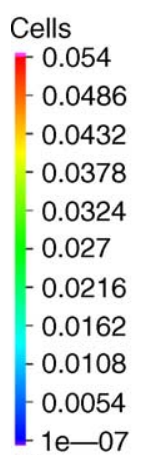

(e)

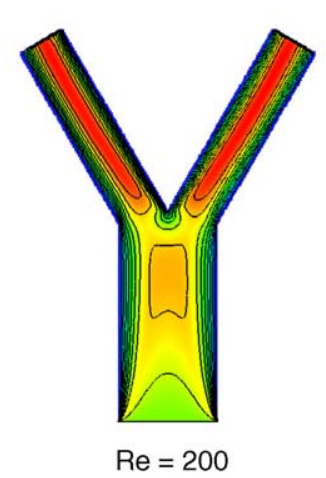

$\mathrm{Re}=200$
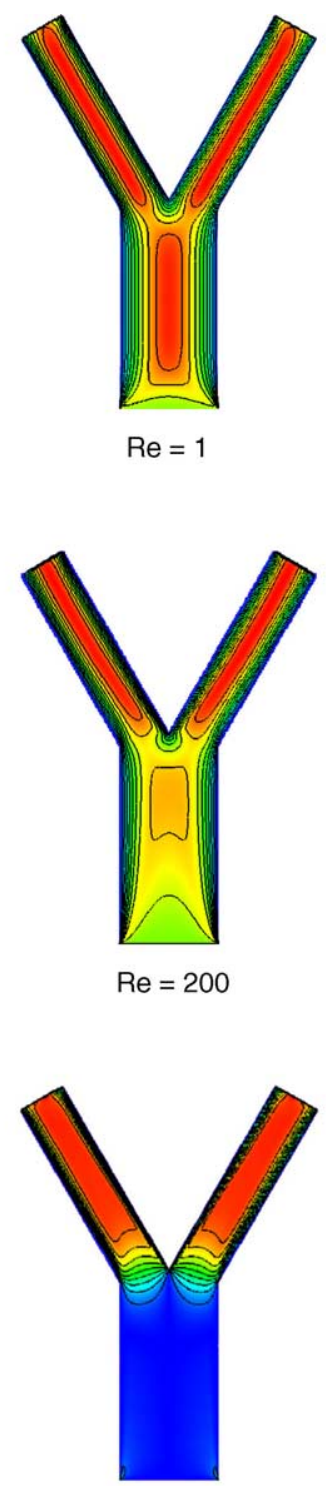

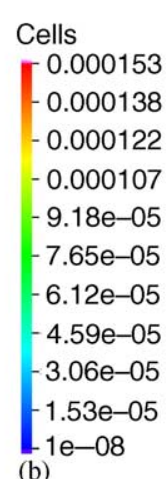

(b)

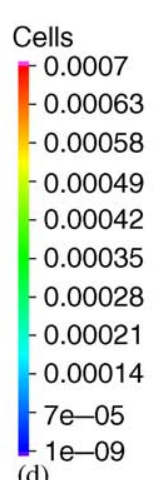

(d)

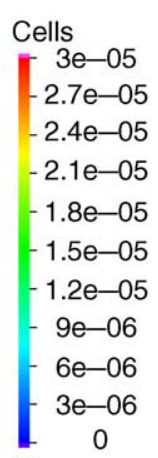

(f)
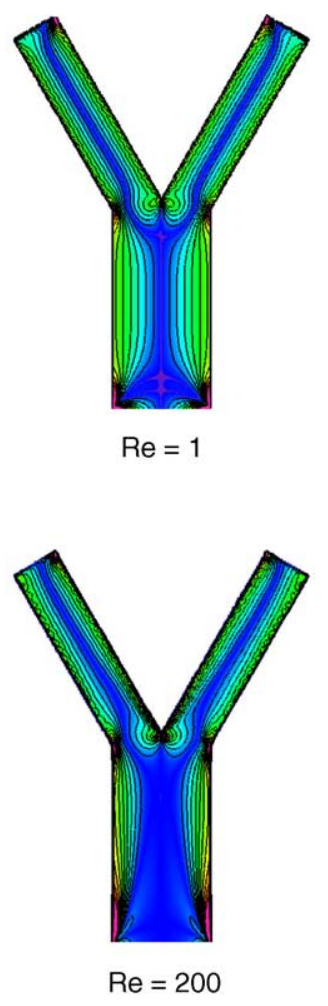

$\mathrm{Re}=1$

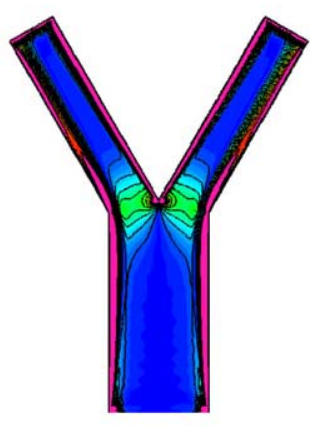

$\mathrm{Re}=1250$

Fig. 2. Contours of velocity magnitudes (left) and shear stress (right) in lattice units for $R e=1(\mathrm{~d} x / \mathrm{d} t=0.0091 \mathrm{~m} / \mathrm{s}), R e=200(\mathrm{~d} x /$ $\mathrm{d} t=0.0912 \mathrm{~m} / \mathrm{s})$ and $R e=1250(\mathrm{~d} x / \mathrm{d} t=0.5706 \mathrm{~m} / \mathrm{s})$.

complex. As the region before the divider is an expansion region, the velocity flow pattern drops before entering the branches. The velocity skews towards the inner walls inside the daughter branches and each of the two streams are bent because of the influence of the secondary motion, with higher velocities near the outer walls of the bend. All these features are in agreement with the literature [5] and with the solutions of the finite volume results, to be presented below.

The $\sigma_{x y}$ components at the inlet are very close to zero, because of the imposed flat velocity profile. The 
corner points $\mathrm{C}$ and $\mathrm{D}$ and the divider region show higher stress values. The stress in the inner walls of the daughter branches is larger than that at the outer walls. As the Reynolds number increases, the shear stress behaves more complex, especially around the divider.

Next, results obtained from the LBM are quantitatively compared to those obtained by a finite volume method [7]. A similar boundary conditions were used. The comparison is made along the centerline $\mathrm{AB}$ and the cross line $\mathrm{CD}$. We have achieved acceptable agreement between the results obtained from the LBM and the results obtained from the finite volume method (FVM) for the two components of the velocity (Fig. 3(a)-(d)). The two methods show that the maxima of $v_{x}$ are shifted towards the outer walls before entering the expansion region and these maxima approach the wall as the Reynolds number increases (since the velocity component increases). As it is shown in Fig. 3(a) for $R e=1$, the maximum difference in the $x$-component of the velocity occurs at the center point which faces the divider for $R e=1$. That is because of the flexibility of the finite volume method in performing local grid refinements at complex regions. For $R e=200$ and 1250, the differences are less than $1 \%$. We also observe that the LBM solution approaches the FVM solution as the grid is refined (see e.g. Fig. 3(a)).

The shear stress component, $\sigma_{x y}$, shows good agreement for both methods, as it is shown in Fig. 3(e)-(g) for the three Reynolds numbers. It is worth noting that the bounce back rule yields good results for the shear stress close to the wall, since $\sigma$ not affected by the constant slip velocity.

While looking at the results along the centerline $\mathrm{AB}$, we have observed good agreement for $v_{x}$ (data not shown). Due to symmetry, $v_{y}$ must be zero along AB. Fig. 3(h) compares the pressure drop along the centerline $\mathrm{AB}$ for the three Reynolds numbers. In this case, discrepancy in the pressure drops has been observed. The maximum difference between the two solutions is about $20 \%$, which occurs at low Reynolds number, near to the divider.

\subsection{Changing geometry}

During vascular surgical planning, we envision that different geometrical solutions need to be tested on the patient anatomical image provided by a suitable imaging technique. Conventionally, with NS solvers, for each newly suggested geometrical solution $S_{N}$, the previous solution $S_{O}$ is discarded, a new grid $G_{N}$ has to be generated and the simulation has to be restarted to obtain the solution $S_{N}$. This may take considerable amount of simulation time. However, there exists some CFD methods which are fully adaptive, such as the finite difference methods. In this section, we present primitive results on adaptivity of lattice Boltzmann method. Given a geometry $G\left(t_{0}\right)$ at time $t_{0}$, we first run the lattice Boltzmann solver towards obtaining the solution $S\left(t_{0}\right)$ while monitoring for a new geometry, not too different from the previous geometry. If at time $t_{i}$ the user introduces a new geometry $G\left(t_{i}\right)$, the simulation instantaneously adapts to the new grid and resumes towards obtaining the solution $S\left(t_{i}\right)$ without a need to restart. The user may end up with a solution $S_{N}$ for the geometry $G\left(t_{N}\right)$. If the lattice Boltzmann method is robust enough, the simulation time from $t_{0}$ to $t_{N}$ could be less than the sum of convergence times $T_{i}^{0}$ for each individual simulation, i.e. $t_{N}-t_{0}<\sum_{i=0}^{N} T_{i}^{0}$. Moreover, the accuracy in $S_{N}$ must be the same as the solution $S_{N}$ (restart) which is obtained by restarting the simulation. This is shown consequently.

We have conducted a number of 2D simulations on the bifurcation benchmark introduced in the previous section, but allowing the bifurcation angle $\theta$ to change during the simulation after equal number of time steps. We have selected this benchmark for its similarity to the planning of a bypass for a diseased artery, where, the surgeon tries different paths to implant the host artery.

The simulation starts at $t_{0}(\theta)$ at $\theta=20^{\circ}$ and the system evolves towards the solution $S(0)$ a number of time steps $i$. At time $t=i$, the angle is increased by $\delta \theta$ and the simulation resumes towards the solution $S(i)$ for the geometry $G(i)$ another $i$ number of time step after which the geometry $G(2 i)$ is introduced and so on, till we end up with $\theta=80^{\circ}$ as our final $G(N)$ geometry. The simulation then converges to the solution $S\left(G_{N}(n i)\right)$. In lattice Boltzmann method, the system converges directly after the mass and momentum reach a given tolerance, chosen to be less than $10^{-5}$ for momentum and less than $10^{-9}$ for mass.

Technically speaking, the initialization and the update of the new geometry are the critical factors 

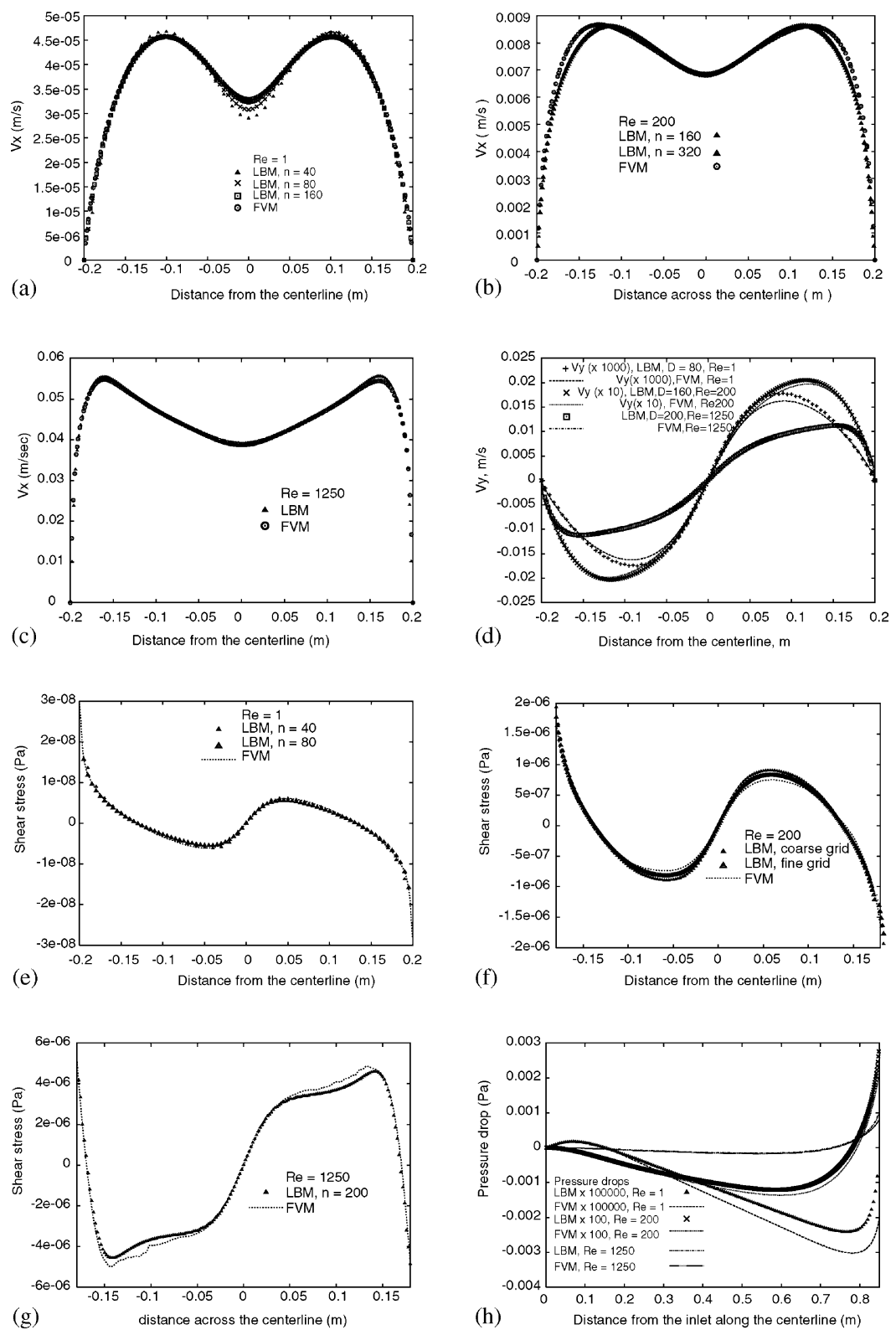

Fig. 3. Comparison of LBM velocity profiles (upper four graphs) and shear stresses (lower left) with the FVM solution along the line CD of the symmetric bifurcation for $R e=1,200$ and 1250 with different grid resolutions. The lower right graph shows the pressure drop along the centerline AB.

which have direct influence on the total simulation time, while the choice of boundary conditions affects both stability and simulation time. In this experiment, we have tested two simple initialization techniques. In both methods, only if the status of a node in the simulation box is changed from fluid to solid or from solid to fluid, the node needs initialization. One way to initialize is to put these nodes to their equilibrium distributions which involves more computational time than the other simpler initialization method such as 


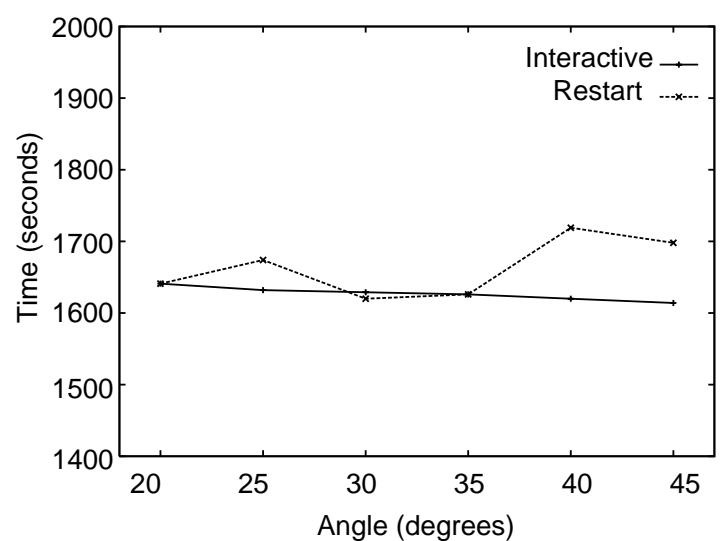

Fig. 4. A lattice Boltzmann comparison between interactive simulations and restarted simulations in terms of simulation time for a symmetric bifurcation benchmark.

assign them to an average value. It is noted that the system forgets about the initialization method in a short transitional time $t_{\text {trans }}$. Fig. 4 shows the total number of nodes, the number of nodes to be updated and the total simulation time for interactive and restarted simulations. As shown in this figure, the total simulation time during an interactively changing geometry is in general smaller than the total simulation time for each individual simulation. This reflects a nice feature of the lattice Boltzmann method and makes it quite suitable for interactive simulation environments. However, the computational gain is less than $10 \%$ in this specific case. Application of acceleration techniques has proven to be feasible [4]. It is noted that other Cartesian grid CFD techniques may share this feature with the lattice Boltzmann method, but the body fitted grid solvers such as the finite element methods will be faced with the time it takes to adapt the new mesh. We are now using the lattice Boltzmann as a core simulation system for an interactive virtual vascular treatment environment using high level architecture (HLA)and a virtual 4D CAVE environment for interaction and visualization $[1,18]$.

\section{Summary}

In this study, we have shown that the lattice Boltzmann method can be used to simulate flow in fixed and changing geometry of common interest to hemody- namics. Steady flow in the symmetric bifurcation has been studied and the results are compared to a finite volume Navier-Stokes solver. The capability of the lattice Boltzmann method for interactive simulations has shortly been demonstrated by studying flow in a changing geometry. It is found that the lattice Boltzmann solver can be an adaptive flow solver without considerable difficulties.

\section{Acknowledgements}

This work is partially funded by the "Steunfonds Soedanese Studenten", Leiden, The Netherlands. We also thank Arjen Berkenbos for performing the finite volume simulations.

\section{References}

[1] R.E. Belleman, J.A. Kaandorp, P.M.A. Sloot, A virtual environment for the exploration of diffusion and flow phenomena in complex geometries, Future Gener. Comput. Syst. 14 (1998) 209-214.

[2] R. Benzi, S. Succi, M. Vergassola, The lattice Boltzmann equation-theory and applications, Phys. Rep. 222 (1992) 145197.

[3] S.A. Berger, L.D. Jou, Flows in stenotic vessels, Ann. Rev. Fluid Mech. 32 (2000) 347-382.

[4] M. Bernaschi, S. Succi, H.D. Chen, R.Y. Zhang, Computing steady state flows with an accelerated lattice Boltzmann technique, Int. J. Mod. Phys. C 13 (2002) 675-687.

[5] C.G. Caro, T.J. Pedley, R.C. Schroter, W.A. Seed, The Mechanics of the Circulation (Oxford University Press, Oxford, 1978).

[6] B. Chopard, M. Droz, Cellular Automata Modeling of Physical Systems, Cambridge University Press (Cambridge, 1998).

[7] FLUENT 4.5, Fluent Inc., 1998. http://www.fluent.com.

[8] I. Foster, C. Kesselmann (Eds.), The Grid: BluePrint for a New Computing Infrastructure, Morgan Kaufmann, Los Altos, CA, 1999.

[9] M. Goyen, M.E. Ladd, J.F. Debatin, J. Barkhausen, K.H. Truemmler, S. Bosk, S.G. Ruehm, Dynamic 3D MR angiography of the pulmonary arteries in under four seconds, J. Mag. Reson. Imaging 13 (2001) 372-377.

[10] M. Krafczyk, M. Cerrolaza, M. Schulz, E. Rank, Analysis of 3D transient blood flow passing through an artificial aortic valve by lattice-Boltzmann methods, J. Biomech. 31 (1998) 453-462.

[11] A.J.C. Ladd, Numerical simulations of particulate suspensions via a discretized Boltzmann equation. Part I. Theoretical foundation, J. Fluid Mech. 271 (1994) 285-309. 
[12] S.E. Maier, D. Meier, P. Boesiger, U. Moser, A. Viele, Human abdominal aorta: comparative measurements of blood flow with MR imaging and multigated Doppler US, Radiology 171 (1989) 487-492.

[13] D.A. McDonald, Blood Flow in Arteries, Camelot Press, 1974.

[14] R.S. Reneman, A.P.G. Hoeks, F.N. van de Vosse, D.N. $\mathrm{Ku}$, Three-dimensional blood flow in bifurcations: computational and experimental analyses and clinical applications, Cerebrovasc. Dis. 10 (1993) 185-192.

[15] S. Succi, The Lattice Boltzmann Equation for Fluid Dynamics and Beyond (Oxford University Press, Oxford, 2001).

[16] C.A. Taylor, T.J.R. Hughes, C.K. Zarins, Finite element modeling of blood flow in arteries, Comp. Meth. Appl. Mech. Eng. 158 (1998) 155-196.

[17] The World Health Report, WHO Publications, 2002.

[18] Z. Zhao, R.G. Belleman, G.D. van Albada, P.M.A. Sloot, AG-IVE: an agent based solution to constructing interactive simulation systems, in: Sloot, et al. (Eds.), Lect. Notes Comput. Sci. 2329 (2002) 693-703.

[19] Q. Zou, S. Hou, S. Chen, G.D. Doolen, An improved incompressible lattice Boltzmann model for time independent flows, J. Stat. Phys. 81 (1995) 35-48.

[20] Q. Zou, X. He, On pressure and velocity boundary conditions for the lattice Boltzmann BGK model, Phys. Fluids 9 (1997) 1591-1598.

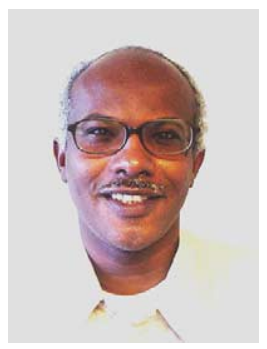

A.M. Artoli graduated in Physics at Assiut University, Egypt, obtained his MSc in Physics at the University of Khartoum, Sudan and received his $\mathrm{PhD}$ in the Section Computational Science, Department of Computer Science at the University of Amsterdam in 2003 on Mesoscopic Computational Haemodynamics. Artoli is an Assistant Professor at the Faculty of Science and Technology, School of Applied Physics, Al Neelain University, Khartoum, Sudan. His current research interests include biofluid computational mechanics.

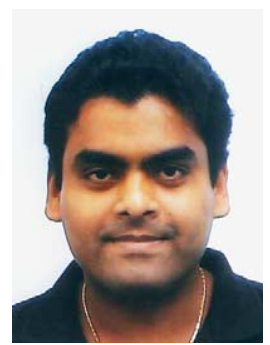

D. Kandhai holds a $\mathrm{PhD}$ in Computational Science from the University of Amsterdam. Currently he is a Post-doc researcher in the Kramers Laboratorium voor Fysische Technologie of Delft University of Technology. His research interests are: transport in porous media, multi-phase flows, lattice-Boltzmann methods, boundary conditions and local grid refinement techniques, and parallel computing.

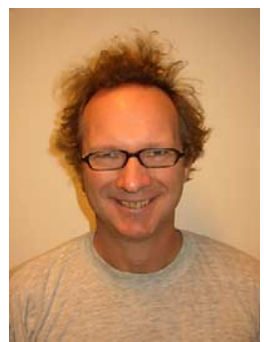

H.C.J. Hoefsloot is an Associate Professor at the University of Amsterdam since 2000. He received his $\mathrm{PhD}$ from Faculty of Sciences, University of Groningen in 1991 on surface tension induced fluid flow. His areas of expertise include mathematics, numerical modeling, data analysis and algorithms.

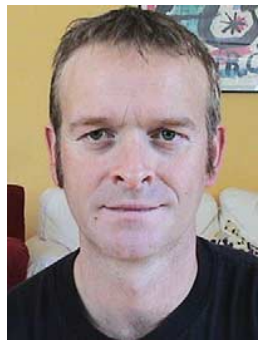

A.G. Hoekstra holds a $\mathrm{PhD}$ in Computational Science from the University of Amsterdam (1994) in the field of parallel and distributed computing. After 2 years of Post-doc research on parallel hierarchical algorithms for many body problems he became assistant professor at the University of Amsterdam, working on parallel and distributed computing and particle based modeling and simulation. Currently he is an associate professor at the University of Amsterdam, with a strong research interest in scientific computing on the Grid and lattice Boltzmann modeling and simulation. He has organized international conferences in the field of High Performance Computing and Computational Science. He has published over a 100 peer reviewed research papers.

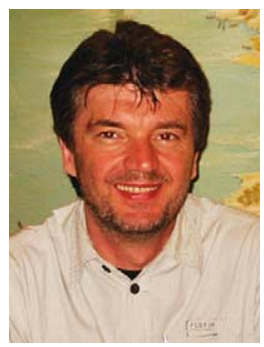

P.M.A. Sloot graduated in physics and chemistry at the University of Amsterdam and worked for 6 years in the Netherlands Cancer Institute as a research assistant. He received his $\mathrm{PhD}$ in computer science in 1988 at the UvA, and visiting professor in various international universities among which Bandung (Indonesia), Saint Petersburg (Russia), and the Santa Fe Institute on Complex Systems (USA). From 1996 to 2001 he was granted a professorship as the NNV distinguished chair on Computational Physics. Since 2002 he is the full professor of Computational Science at the UvA. He has initiated over eight EU research and development projects in ICT, is co-leading the EU Cross-Grid project and one of the board members of the EU Health-Grid Initiative. Over the last 10 years he has been in a large number of national and international committees on High Performance Computing. With Jack Dongarra he is responsible for the International Conferences on Computational Science Series. He has been elected by the European Science foundation as the chair of the Problem Solving Environment Programme. Peter Sloot has published over 200 peer reviewed papers and books on various topics in computer science and guided over $10 \mathrm{PhD}$ theses. 